\title{
Physical Basis of the Controlled Electrochemical Treatment of Soils from Oil Products
}

\author{
PROSTOV Sergey M., GUCAL Maxim B., SHABANOV Evgeniy F. \\ Mining Institute, T.F. Gorbachev Kuzbass State Technical University, 650000, \\ Kemerovo, Russia,
}

\begin{abstract}
There are the main sources of pollution of soils Kuzbass are presented. The ranges of electrical resistivity of solutions containing oil and contaminated soil, depending on the degree of contamination are presented
\end{abstract}

Keywords: specific electric resistivity, electrochemical treatment, control, oil products.

\section{Introduction}

Currently the problem of soil clearing from various pollutants of industrial origin becoming increasingly urgent. The most contaminated soils are in populated areas near highways, on the territories of the industrial facilities, both operation and abandon. Just a serious problem is the contamination of soil by radioactive elements as a result of the nuclear industry. Also rather serious problem is contaminating of soils as a result of activity of atom industry. As ecotoxicants can be heavy metals, oil and oil products, cyanides, and organic chlorine compounds. Concentration of toxicants can exceed maximum allowable concentration (MAC) in hundred times.

In Kemerovo region general environmental conditions are defined by development industry and the predominance of the urban population. Industrial production is the leading sector of the region economy with a predominance of the following industries in the regional economy: ferrous metallurgy, coal and chemical industry. In the region there is a great number of boilers operating on solid fuels i.e. coal. Products of coal combustion (slag and ash) are stored and kept on the ground surface what leads to contamination by the chemical elements contained in the ash and slag.

There are 331 operating wastewater treatment facilities in the region, including 164 industrial wastewater treatment facilities, 20 storm-water runoff treatment facilities and 147 household sewage and mixed one with sewage. The capacity of the treatment facilities, after which the wastewater is discharged into surface water bodies is over 760 million cubic meters.

It seems that a problem of development of the controlled methods for purification of soil pollutants without prior removal of the contaminated soil is urgent. Monitoring of the property changes of the treated area allows timely adjustments to operating practices and optimization of costs. In particular, the intensity of the electrochemical treatment is controlled by electric current density, and in case of combination of the electrochemical process with biological one, they control temperature of the processing zone. To increase the effectiveness of the treatment methods it's necessary to develop efficient and not so efficient ways of geocontrol providing definition of the geometrical parameters and substantiation of the optimal treatment modes for a zone under treatment. To meet those challenges to the greatest extent it's required to develop geo-electric methods 
of control, based on the parameters of the electric fields due to the physical properties and composition of soils.

\section{Work description}

The Kuzbass coal industry presents 62 underground mines, 57 open pits and 49 preparation plants and units. Their production capacity is 245 million tons of coal per year in coal mining and 166 million tons in processing.

The main sources of soil contamination at the mining and processing sites in the
Kuzbass region are:

- Facilities for industrial wastewater and sewage (groundwater filters);

- Fuel stations;

- Boilers and power plants;

- Dry and slurry dumps;

- Sludge pits of preparation plants.

The number of objects, the sources of soil contamination, in one of the largest coal producers of OJSC UK'Kuzbassrazrezu gol' are shown in Table 1.

Table 1: Sources of pollution in the OJSC UK'Kuzbassrazrezugol'

\begin{tabular}{|c|c|c|c|c|c|c|c|c|}
\hline & \multirow{2}{*}{$\begin{array}{c}\text { Oil and } \\
\text { lubricant } \\
\text { Oarehou- } \\
\text { se }\end{array}$} & \multirow{2}{*}{$\begin{array}{c}\text { Bo } \\
\text { il- } \\
\text { er }\end{array}$} & $\begin{array}{c}\text { Prepar- } \\
\text { ation }\end{array}$ & $\begin{array}{c}\text { Groundwater- } \\
\text { filters }\end{array}$ & $\begin{array}{c}\text { Slud } \\
\text {-ge } \\
\text { pits }\end{array}$ & $\begin{array}{c}\text { Comp- } \\
\text { Slurry } \\
\text { pits } \\
\text { lexes }\end{array}$ & & $\begin{array}{c}\text { Dry } \\
\text { dum } \\
\text {-ped }\end{array}$ \\
\hline Kedrovskiy & 1 & 1 & 1 & 2 & 3 & 2 & 1 & 4 \\
\hline Mokhovskiy & 3 & 3 & - & 2 & 1 & 2 & 2 & 7 \\
\hline Bachatskiy & 2 & 1 & 2 & 1 & - & 2 & - & 4 \\
\hline Krasnobrodskiy & 3 & 5 & 1 & 2 & 2 & 2 & - & 9 \\
\hline Taldinskiy & 3 & 3 & - & 1 & 1 & 1 & 1 & 11 \\
\hline Kaltanskiy & 2 & 2 & - & - & 5 & 2 & - & 7 \\
\hline $\begin{array}{c}\text { OJSC } \\
\text { UK'Kuzbassr- } \\
\text { azrezugol' } \\
\text { OAO }\end{array}$ & 14 & 15 & 4 & 8 & 12 & 11 & 4 & 42 \\
\hline
\end{tabular}

The electrochemical method is a potentially promising treatment method out of the existing methods of cleaning soil from industrial pollutants (Fig.1). A distinctive feature of this method that it can be used for cleaning soils with low filtration capacity on-situ, without a notch and moving of soil.

The physical basis of this method is the electro osmotic movement of ecotoxicants under the HelmholtzSmoluchowski equation:

$$
\bar{V}=-\frac{\varepsilon \varsigma}{\mu} \operatorname{grad} \varphi
$$

where $\bar{V}$ - average capillary flow rate as per the cross section, $\mathrm{m} / \mathrm{s} ; \varepsilon$ - total dialectical permeability, $\mathrm{F} / \mathrm{m} ; \mu$ dynamic coefficient of viscosity, $\mathrm{Pa} \cdot \mathrm{s} ; \zeta$ - electric kinetic potential, V; $\varphi$ potential of outer electric field, V.

On the basis of the dimensional analysis, the dependence of the effective rate for the module $V_{э \phi}$ (rate of flow) was obtained:

$$
V_{щ T}=C \sigma_{0} \frac{m^{2} R}{\mu} E=\frac{m \varsigma \varepsilon}{\mu} E=K_{m} E,
$$


where $\mathrm{C}$ - constant; $\sigma_{0}$ - specific volume of charge density of the ions of diffuse layer, $\mathrm{C} / \mathrm{m}^{3} ; m-$ soil porosity; $R-$ hydraulic radius of pores, $\mathrm{m} ; K_{\ni}$ coefficient of electro osmotic activity, $\mathrm{m}^{2} /(\mathrm{V} \cdot \mathrm{s}) ; \mathrm{E}$ - field density, $\mathrm{V} / \mathrm{m}$.

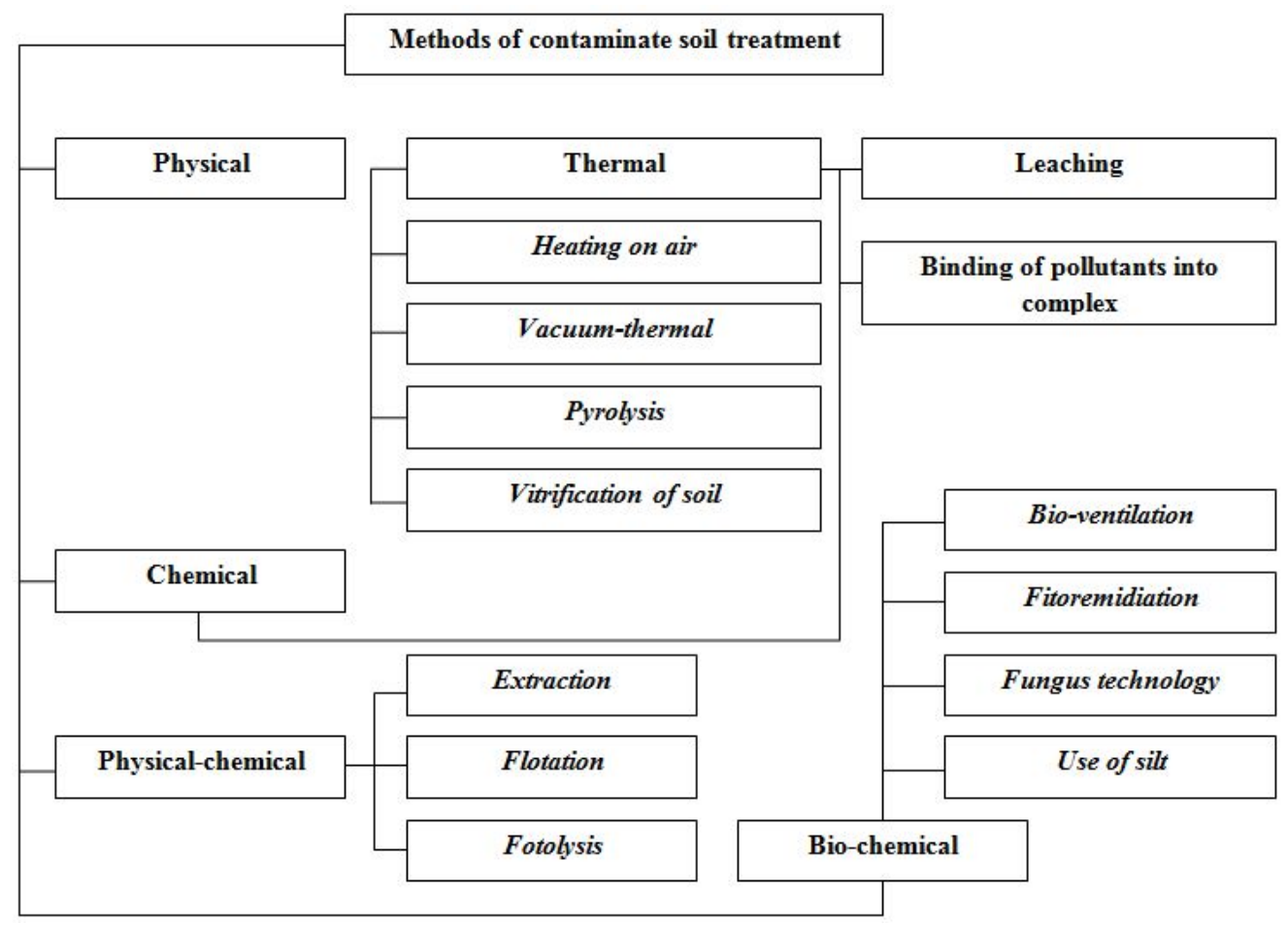

Fig. 1:Classification of contaminated soil treatment methods

During cleaning the pollutants move along the electric field lines, the structure of which is determined by the location of the electrodes; the rate of pollutants' movement depends on the field intensity, allowing monitoring the cleaning process and managing it. Initial concentrations of eco-toxicants can be reduced from 10-50 $\mathrm{mg} / \mathrm{kg}$ to $1.10 \mathrm{mg} / \mathrm{kg}$, which is well within the existing norms.

The main test parameters of the geoelectric method are: specific electrical resistivity (SER), dielectric and magnetic permeability, electric strength, potentials of steady and unsteady natural electric fields.

Effective SER of the moisture saturated rock, which is a three-phase medium, is descried by the empirical dependence ${ }^{[1]}$ :

$$
\rho_{\breve{U}}=\frac{\alpha \kappa_{O}}{m^{\beta} W^{\gamma}} \rho_{\bar{b}},
$$

where $\rho_{\breve{И}}$ - porosity (pore porosity); $W$ - moisture saturation coefficient of pores and cracks; $\rho_{\bar{b}}-\mathrm{SER}$ of the solution filling the pore space, $\Omega \cdot \mathrm{m} ; \kappa_{O}$, $\alpha, \beta, \gamma-$ empirical parameters depended on the structural and textural features of the studied soils (rocks); $\kappa_{O^{-}}$ parameter that takes into account a surface conductivity of clay micro-layer on the pore surface; $\alpha$ - parameter depending on the type of geological deposits; $\beta-$ parameter defined by structure of pore structure (mainly by sinuous channels); $\gamma \quad-$ parameter 
depending on wettability of the pore surface by solution.

Table 2: Predictive values of structural parameters $\beta$ и $\gamma$

\begin{tabular}{|c|c|c|c|c|c|c|c|c|c|c|}
\hline \multirow{2}{*}{\multicolumn{2}{|c|}{ Type of soil }} & \multicolumn{9}{|c|}{ Parameter $\beta$} \\
\hline & & \multicolumn{3}{|c|}{$\begin{array}{l}\frac{\text { Sand clay }}{\bar{m}}=0,314 \\
\bar{W}=0,187)\end{array}$} & \multicolumn{3}{|c|}{$\begin{array}{l}\frac{\text { Clay loam }}{(\bar{m}}=0,296 ; \\
\bar{W}=0,227)\end{array}$} & \multicolumn{3}{|c|}{$\begin{aligned} & \text { Clay } \\
(\bar{m} & =0,324 \\
\bar{W} & =0,189)\end{aligned}$} \\
\hline \multicolumn{2}{|c|}{ Parameter $\gamma$} & 1,8 & 2,2 & 2,6 & 1,8 & 2,2 & 2,6 & 1,8 & 2,2 & 2,6 \\
\hline \multirow{5}{*}{$\frac{\rho_{\breve{U}}}{\rho_{\bar{L}}}$} & 10,0 & 0,59 & 1,15 & 1,72 & 0,30 & 0,79 & 1,27 & 0,62 & 1,21 & 1,80 \\
\hline & 5,0 & 1,18 & 1,74 & 2,32 & 0,87 & 1,36 & 1,84 & 1,23 & 1,82 & 2,41 \\
\hline & 2,0 & 1,96 & 2,51 & 3,13 & 1,62 & 2,11 & 2,60 & 2,05 & 2,64 & 3,23 \\
\hline & 1,5 & 2,20 & 2,77 & 3,34 & 1,69 & 2,35 & 2,83 & 2,30 & 2,89 & 3,84 \\
\hline & 1,2 & 2,40 & 2,95 & 3,53 & 2,04 & 2,53 & 3,02 & 2,50 & 3,09 & 4,46 \\
\hline
\end{tabular}

Table 2 presents estimated values of the parameters $\beta$ and $\gamma$, obtained due to the use of the true range $\rho_{\breve{M}} / \rho_{\bar{b}}$ at geocontrol of the wetted rocks, as well as average values of physical and technical parameters $\bar{m}$ and $\bar{W}$ for clay ground of Kuzbass, given in the article [2], for the major types of clay-bearing soils.

For further analysis of the process of electrochemical treatment of the contaminated soils the information regarding electro physical and electrochemical properties of natural water solutions and liquids containing contaminants saturated soils is required. An experimental study of the electrical properties of electrolyte solutions containing oil, and measurement at a constant and alternating electric current is carried out.

Fig. 2 presents measurements of specific electrical resistance (SER) $\rho_{u}$ of $\mathrm{NaCl}$ solution depending on the concentration of the salt in the solution, and the AC frequency; and Fig. 3 presents similar SER $\rho_{M}$ dependences of motor oil and gasoline. From these data, it' clear that that all oil products are electrically very contrast because their SER 3 times higher, at least, than resistivity of natural water solutions.

Since the formation of stable, homogeneous oil-water slurry is very difficult, for further theoretical estimation, a logarithmic dependence of the average for the two-component medium, allowing calculation of the required resistivity with higher accuracy ${ }^{[3]}$ was used:

$$
\lg \rho_{\sigma}=V_{M} \lg \rho_{H}+V_{u} \lg \rho_{u},
$$

where $\rho_{\sigma}, \rho_{H}, \rho_{u}$ - SER accordingly weighted, oil and electrolyte; $V_{M}, V_{u}-$ volume of oil and the electrolyte in the solution, respectively.

Substituting the data from Fig. 2, 3 into equation (4), the dependencies on the average weighted values $\rho_{\sigma}$ of the oil content $V_{\mathcal{M}} / V_{u}$ for DC (Fig. 4) and AC (Fig. 5) currents were obtained. 


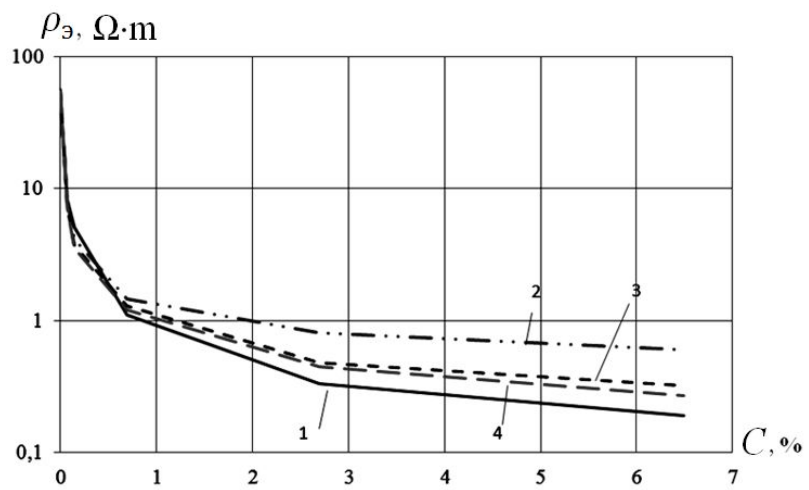

1 -direct current (DC);2 - alternating current (AC), $f=10 \mathrm{~Hz} ; 3-200 \mathrm{~Hz} ; 4-1000 \mathrm{~Hz}$

Fig. 2. Dependence of SER solution $\rho_{u}$ from salt $\mathrm{C}$ concentration in solution

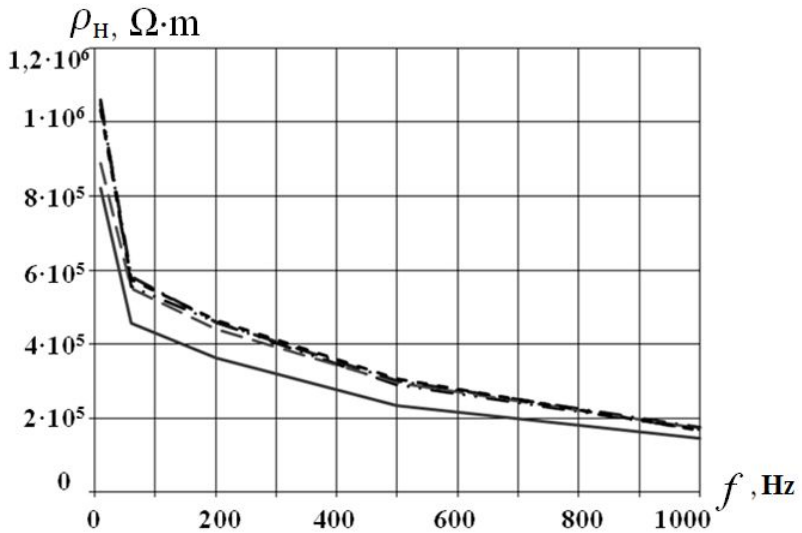

- G-Energy F Synth 0W-40; — — - Mobil Synt S 5W-40; _ _ - - Shell Helix Ultra (worked out);_ _. - - Shell Helix Ultra; - gasoline Ai-92

Fig. 3: Dependence of SER $\rho_{M}$ of motor oil from frequency of alternative current

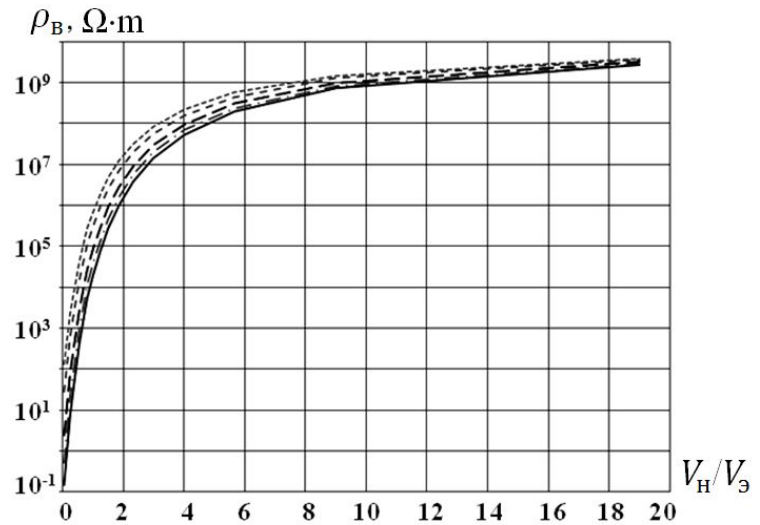

saturated solution; - - - salt concentration in solution $\mathrm{C}=50 \mathrm{~g} / \mathrm{l}$;

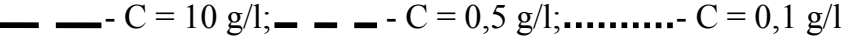

Fig. 4: Dependence of the average weighted values $\rho_{\sigma}$ from relatively to the content of oil products on DC 


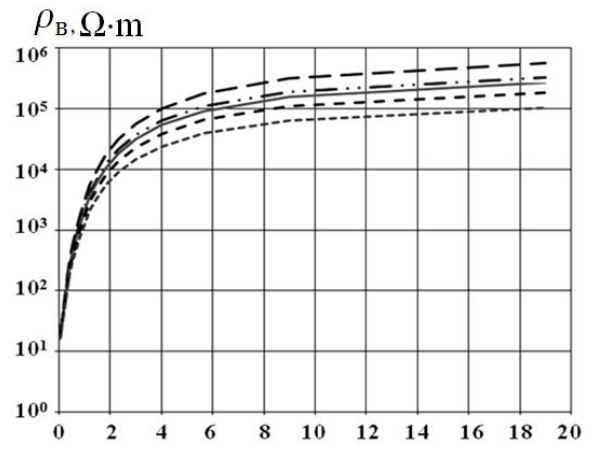

(a)

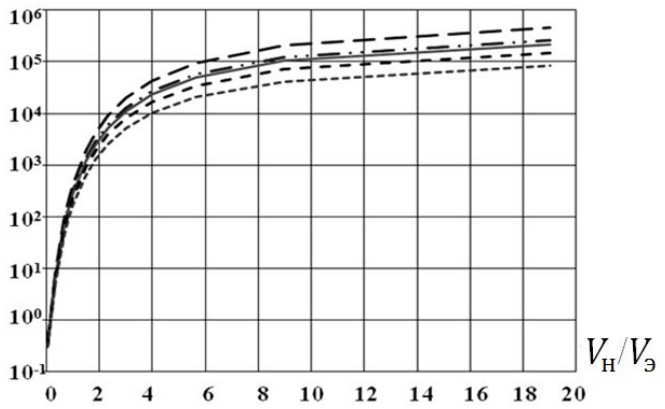

(b) $200 \mathrm{~Hz} ;$ - - - - $500 \mathrm{~Hz}$ $1000 \mathrm{~Hz}$

Fig. 5: SER average weighted depending on the frequency of the alternating current and salt concentration in electrolyte $\mathrm{C}=0,5 \mathrm{~g} / 1$ (a); $50 \mathrm{~g} / 1$ (b)

To implement the theoretical dependence (3) following assumptions were made. According to studies of V.N. Kobranovoy ${ }^{[4]}$ a parameter value $\alpha$ can be taken as 1 for sand and clay deposits of the same type. Parameter $\kappa_{O}$ of the surface conductivity can also assumed as 1 , because if contaminated by oil products is likely to form oil film of littleconductivity on the surface of the solid particles of the soil.

Given the ranges of the structural parameters $\beta$ and $\gamma$ shown in Table. 2, the dependence of the efficient SER changes of the main clay of the Quaternary deposits in Kuzbass were calculated. They are partially shown on Fig. 6.

These given dependences allowed forming a database for interpretation of the experimental sounding if the relation $V_{M} / V_{u}$ changes at monitoring as per the value $\rho_{\breve{\Pi}}$.

\section{Conclusion}

The analysis of the results led to the following conclusions.

1. SER of natural liquids and oily pollutants saturating soil, are electrically contrasting, and several times different in the direction of the contaminant, which allows to determine sufficiently the geometrical parameters of the zone of the contaminated soil and the degree of its contamination.

2. For the diagnosis of geometrical parameters of the contaminated areas and control over spreading of the eco-toxicant, it's expedient to develop methods of electrical and geo-electric exploration on direct and alternating current, which improves the accuracy of control, and let apply contact, contactless and boreholefree methods of measurements.

3. Defined dependences and ranges of the changes of electro-physical parameters of rocks and soils, depending on SER of the pollutant solution allow us to develop methods and techniques to control the process of electrochemical contaminated soils treatment. 


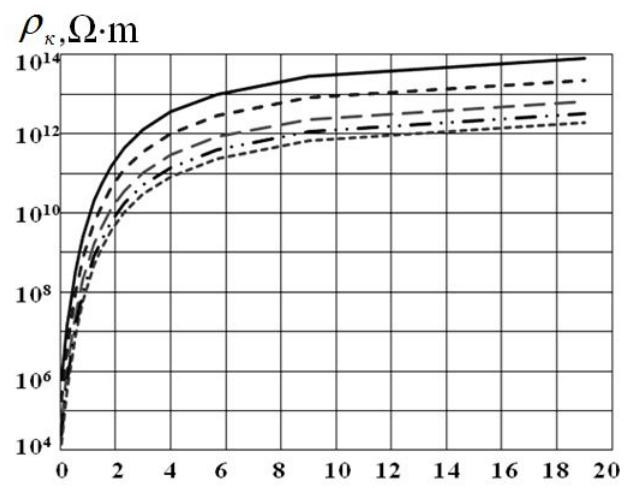

(a)

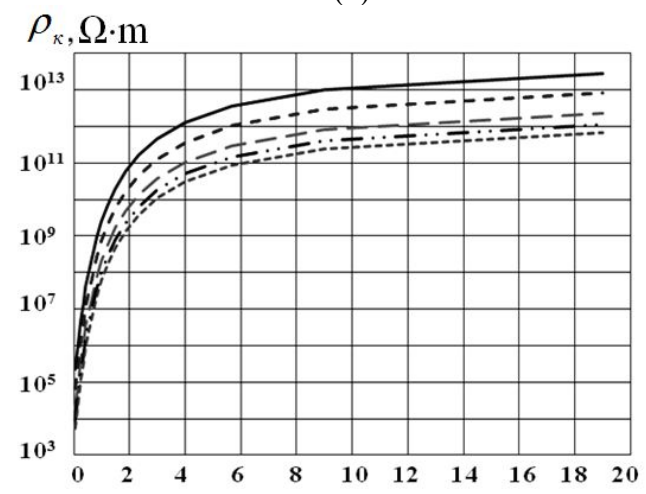

(c)

$\rho_{\kappa}, \Omega \cdot \mathrm{m}$

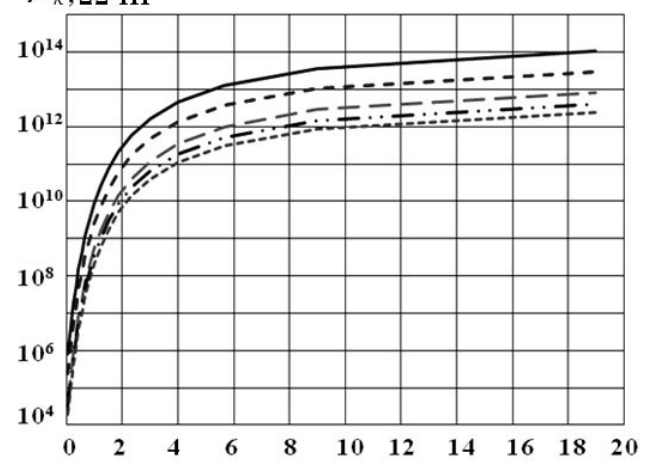

(e)

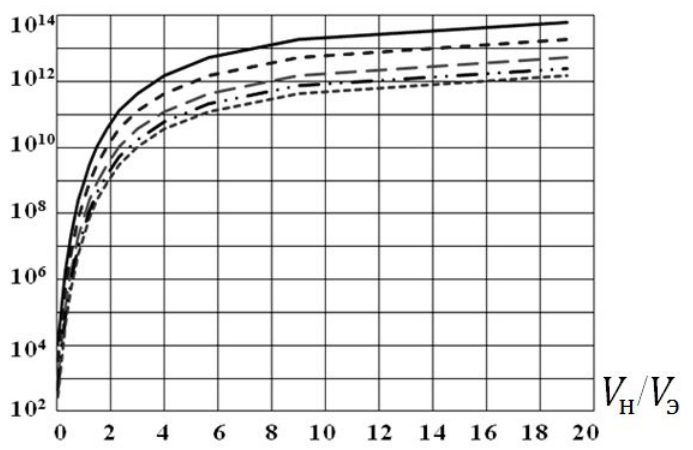

(b)

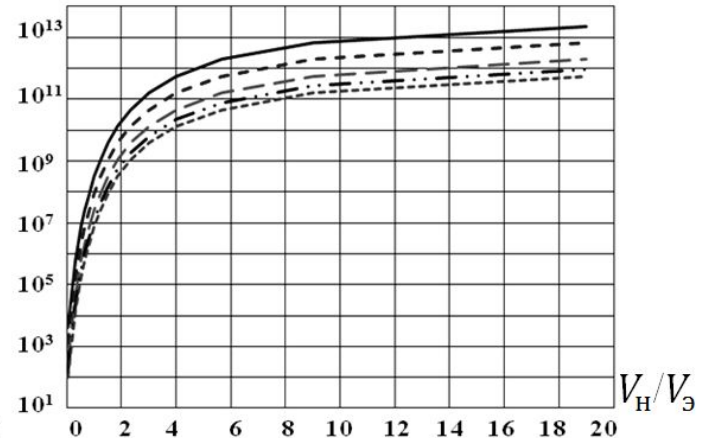

(d)

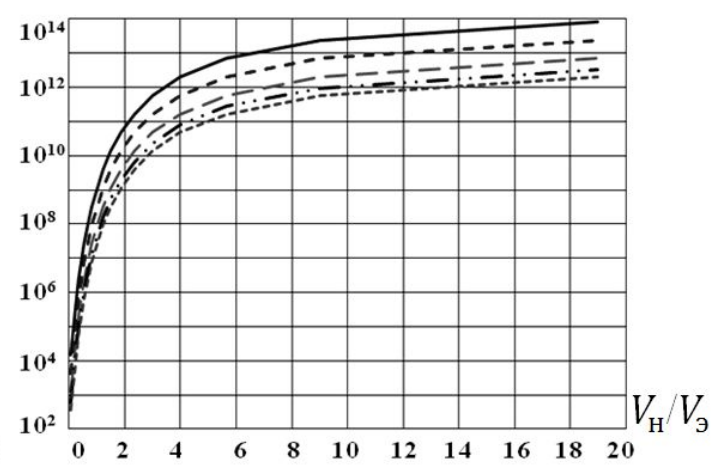

(f)

Fig.6:Dependence the of the efficient SER changes $\rho_{\breve{U}}$ of Kuzbass soils from moisture saturation coefficient of pores and cracks $\mathrm{W}$, relative content of oil in average weighted $V_{M} / V_{u}$ and salt concentration in electrolyte $\mathrm{C}, \mathrm{g} / \mathrm{l}$ : sand clay $-C=0,5$ (a), $C=50$ (b); clay loam - $C=0,5$ (c), $C=50$ (d); clay $-C=0,5$ (e), $C=50$ (f):

\section{References}

[1] Prostov S.M. Geo-electrical control of strengthen clay rocks zones / SM Simple, VA Haymaylyainen, MV Gutsal, SP
Bakhaeva; RANS. - Tomsk Univ.

University Press, 2005. - 127 p. 
[2] Stumpf, G.G. Physical and technical properties of rocks and coals of Kuznetsk Basin / G.G. Stumpf, A. Ryzhkov, VA Shalamanov, A.I. Petrov. - Moscow.: Nedra, 1994. - 447 p.

[3] Rzhevskiy V.V. Basic physics of rocks: the textbook for high schools. 4th- ed., Rev. and add. / V.V. Rzhevskiy G.Y. Novik. - Moscow.: Nedra, 1984. - 359 p. [4] Kobranova V.N. Physical properties of rocks. - Moscow.: Gos. scientific and engineering. ed of oil and mining fuel literature, 1962. - 490p. 